\title{
Asymptotic behavior for nonlinear Schrödinger equations with critical time-decaying harmonic potential.
}

\section{by Masaki Kawamoto}

Department of Engineering for Production, Graduate School of Science and Engineering, Ehime University, 3 Bunkyo-cho Matsuyama, Ehime, 790-8577. Japan

Email: kawamoto.masaki.zs@ehime-u.ac.jp

\begin{abstract}
Time-decaying harmonic oscillators yield dispersive estimates with weak decay, and change the threshold power of the nonlinearity between the short and the long range. In the non-critical case for the time-decaying harmonic oscillator, this threshold can be characterized by polynomial nonlinearities. However, in the critical case, it is difficult to characterize the threshold using only polynomial terms, and thus we use logarithmic nonlinear terms.
\end{abstract}

Keywords; Nonlinear scattering theory; Long-range scattering; Time-dependent harmonic oscillators; Time-dependent magnetic fields

MSC; primary 35Q55, secondly 35J10

\section{Introduction}

In this study, we consider nonlinear Schrödinger (NLS) equations with time-dependent harmonic potentials:

$$
\left\{\begin{array}{l}
i \partial_{t} u(t, x)-\left(-\Delta / 2+\sigma(t)|x|^{2} / 2\right) u(t, x)=F(u(t, x)) u(t, x), \\
u(0, x)=u_{0}(x)
\end{array}\right.
$$

where $(t, x) \in \mathbf{R} \times \mathbf{R}^{n}, n \in\{1,2,3\}$, and $F: \mathbf{C} \rightarrow \mathbf{R}$ is a nonlinear term to be defined later. Let

$$
H_{0}(t):=-\Delta / 2+\sigma(t)|x|^{2} / 2
$$

$\sigma(t)|x|^{2} / 2$ is called time-dependent harmonic potential with coefficient $\sigma(t)$. We make the following assumption on $\sigma(t) \in L^{\infty}\left(\mathbf{R}_{t}\right)$.

Assumption 1.1. Let $\zeta_{1}(t)$ and $\zeta_{2}(t)$ be the fundamental solutions to the following equations:

$$
\zeta_{j}^{\prime \prime}(t)+\sigma(t) \zeta_{j}(t)=0, \quad\left\{\begin{array} { l } 
{ \zeta _ { 1 } ( 0 ) = 1 , } \\
{ \zeta _ { 1 } ^ { \prime } ( 0 ) = 0 , }
\end{array} \quad \left\{\begin{array}{l}
\zeta_{2}(0)=0, \\
\zeta_{2}^{\prime}(0)=1 .
\end{array}\right.\right.
$$

Then, there exist $c>0, r_{0} \gg 1$, and $c_{1, \pm}, c_{2, \pm} \notin\{0, \infty,-\infty\}$ such that for all $|t|>r_{0}$, we have

$$
\left|\zeta_{2}(t)\right| \geq c
$$

and

$$
\lim _{t \rightarrow \pm \infty} \frac{\zeta_{1}(t)}{|t|^{1 / 2}}=c_{1, \pm}, \quad \lim _{t \rightarrow \pm \infty} \frac{\zeta_{2}(t)}{|t|^{1 / 2} \log |t|}=c_{2, \pm} .
$$

Moreover, $\zeta_{1}(t), \zeta_{2}(t), \zeta_{1}^{\prime}(t)$, and $\zeta_{2}^{\prime}(t)$ are continuous functions in $t$. 
Remark 1.2. For simplicity, let us redefine $\sigma$ as

$$
\sigma(t)= \begin{cases}\sigma_{s}(t), & |t| \leq r_{0} \\ 1 /\left(4 t^{2}\right), & |t|>r_{0}\end{cases}
$$

where $\sigma_{s}(t)$ is a bounded smooth function. Then, for $|t| \geq r_{0}$, the functions $y_{1}(t)=|t|^{1 / 2}$ and $y_{2}(t)=|t|^{1 / 2} \log |t|$ are the linearly independent solutions to $y^{\prime \prime}(t)+\sigma(t) y(t)=0$. Hence, in (4), by writing $\zeta_{1}(t)=c_{1,1, \pm} y_{1}(t)+c_{1,2, \pm} y_{2}(t)$ and $\zeta_{2}(t)=c_{2,1, \pm} y_{1}(t)+c_{2,2, \pm} y_{2}(t)$, Assumption 1.1 implies $c_{1,1, \pm}=c_{1, \pm}, c_{1,2, \pm}=0, c_{2,1, \pm} \in \mathbf{R}$, and $c_{2,2, \pm}=c_{2, \pm}$. We provide examples of $\sigma(t)$ satisfying Assumption 1.1 in $\S 4$.

We now let $U_{0}(t, s)$ be a propagator for $H_{0}(t)$, that is, a family of unitary operators $\left\{U_{0}(t, s)\right\}_{(t, s) \in \mathbf{R}^{2}}$ on $L^{2}\left(\mathbf{R}^{n}\right)$ such that for all $t, s, \tau \in \mathbf{R}$, we have

$$
\begin{aligned}
& i \partial_{t} U_{0}(t, s)=H_{0}(t) U_{0}(t, s), \quad i \partial_{s} U_{0}(t, s)=-U_{0}(t, s) H_{0}(s), \\
& U_{0}(t, \tau) U_{0}(\tau, s)=U_{0}(t, s), \quad U_{0}(s, s)=\operatorname{Id}_{L^{2}\left(\mathbf{R}^{n}\right)}, \quad U_{0}(t, s) \mathscr{D}\left(H_{0}(s)\right) \subset \mathscr{D}\left(H_{0}(s)\right)
\end{aligned}
$$

on $\mathscr{D}\left(H_{0}(s)\right)$. By using $\zeta_{1}(t)$ and $\zeta_{2}(t)$, the following MDFM decomposition can be obtained, see Korotyaev [14] (also Carles [2], Kawamoto [1], and Kawamoto-Muramatsu [13]).

Lemma 1.3. For $\phi \in \mathscr{S}\left(\mathbf{R}^{n}\right)$, let

$$
(\mathcal{M}(\tau) \phi)(x)=e^{i x^{2} /(2 \tau)} \phi(x), \quad(\mathcal{D}(\tau) \phi)(x)=\frac{1}{(i \tau)^{n / 2}} \phi(x / \tau) .
$$

Then, the following MDFM decomposition holds:

$$
U_{0}(t, 0)=\mathcal{M}\left(\frac{\zeta_{2}(t)}{\zeta_{2}^{\prime}(t)}\right) \mathcal{D}\left(\zeta_{2}(t)\right) \mathscr{F} \mathcal{M}\left(\frac{\zeta_{2}(t)}{\zeta_{1}(t)}\right)
$$

By the decomposition formula in [14], we have the following dispersive estimates: For $\phi \in L^{1}\left(\mathbf{R}^{n}\right)$,

$$
\left\|U_{0}(t, 0) \phi\right\|_{\infty} \leq C\left|\zeta_{2}(t)\right|^{-n / 2}\|\phi\|_{1}
$$

and

$$
\left\|U_{0}(t, s) \phi\right\|_{\infty} \leq C\left|\zeta_{1}(s) \zeta_{2}(t)-\zeta_{1}(t) \zeta_{2}(s)\right|^{-n / 2}\|\phi\|_{1},
$$

where $\|\cdot\|_{q}, 1 \leq q \leq \infty$, denotes the norm on $L^{q}\left(\mathbf{R}^{n}\right)$. Hence, we immediately obtain the following proposition.

Proposition 1.4. Under Assumption 1.1, for all $|t|>r_{0}$, there exists $C>0$ such that

$$
\left\|U_{0}(t, 0) \phi\right\|_{\infty} \leq C|t|^{-n / 4}(\log |t|)^{-n / 2}\|\phi\|_{1} .
$$

We now introduce the definition of nonlinearity $F$. Consider the case where $\sigma(t) \equiv 0$ and $F(u(t, x))=|u(t, x)|^{\theta}$ for some $\theta>0$. According to studies by Strauss [22], Barab [1], TsutsumiYajima [23], and so on, there exists $u_{ \pm} \in L^{2}\left(\mathbf{R}^{n}\right)$ such that

$$
\lim _{t \rightarrow \pm \infty}\left\|u(t, \cdot)-e^{i t \Delta / 2} u_{ \pm}\right\|_{2}=0
$$

holds for $2 / n<\theta$ and fails for $0<\theta \leq 2 / n$. Therefore, in this sense, when $\theta>2 / n$ we say the nonlinearity is short-range, when $\theta \leq 2 / n$ we say the nonlinearity is long-range, and we say the power $\theta=2 / n$ a threshold. 
Next, we consider the case where $\sigma(t)=\sigma_{1} t^{-2}$ with $\sigma_{1} \in(0,1 / 4)$. In Kawamoto-Yoneyama [12], it was shown that the decay rate of the $L^{\infty}-L^{1}$ dispersive estimate for free solution is of order $\mathcal{O}\left(t^{-\theta_{1}}\right), \theta_{1}=n\left(1+\sqrt{1-4 \sigma_{1}}\right) / 4$, then the power-type nonlinearity $|u|^{\theta_{2}} u$ is included in the short-range class for $\theta_{1} \theta_{2}>1$, whereas it is included in the long-range class for $\theta_{1} \theta_{2}=1$ (see [13]). The key for this characterization of the threshold is the convergence and divergence of the following improper integral

$$
\int_{1}^{\infty}\|F(u(s, \cdot))\|_{\infty} d s=\int_{1}^{\infty}\|u(s, \cdot)\|_{\infty}^{\theta_{2}} d s .
$$

Imitating the approach by Hayashi-Naumkin [7, we can show that $\|u(s, \cdot)\|_{\infty} \leq C|s|^{-\theta_{1}}$ under the suitable conditions. Then we notice that if $\theta_{1} \theta_{2}>1$, (6) converges and otherwise it may diverge. Consequently, imitating the approach by [7], we can show that if (6) converges, then the solution $u(t, x)$ converges to free solution (i.e., $\theta_{2}>1 / \theta_{1}$ is in short-range class) and if $\theta_{2}=1 / \theta_{1}$, then $u(t, x)$ never converges to free solution (i.e., $\theta_{2}=1 / \theta_{1}$ is a threshold).

Now back to our model. Noting (5) and following the previous method, the critical nonlinearity can be determined through the divergence and convergence of

$$
\int_{1}^{\infty}\left|F\left(|s|^{-n / 4}(\log |s|)^{-n / 2}\right)\right| d s .
$$

If we put $F(u)=|u|^{\theta_{3}}$. Then (7) is equivalent to

$$
\int_{1}^{\infty} \frac{1}{s^{n \theta_{3} / 4}(\log s)^{n \theta_{3} / 2}} d s
$$

By virtue of log-decay term in (5), improper integral (8) is clearly integrable for $\theta_{3}=4 / n$, and hence it can be expected that $4 / n$ is in the short-range. On the other hand, for any $\theta_{3}<4 / n$, improper integral (88) diverges, and hence to determine the threshold for this model is difficult with only using polynomial type nonlinearities. To obtain a threshold to this model, we shall give log-modification to nonlinearity $F(u)$. Putting $F(u)=|u|^{4 / n}(\log (1+1 /|u|))^{\theta_{4}}$, (7) is estimated as

$$
\int_{1}^{\infty}\left(\log \left(1+s^{n / 4}(\log s)^{n / 2}\right)\right)^{\theta_{4}} \frac{d s}{s(\log s)^{2}} \sim \int_{1}^{\infty} \frac{d s}{s(\log s)^{2-\theta_{4}}}
$$

and thus the following characterization of nonlinearities is more suitable for this model.

Assumption 1.5. Let $F(u)=F_{L}(u)+F_{S}(u)$, where

$$
F_{S}(u)=\mu_{S}|u|^{4 / n}\left(\log \left(R+\frac{1}{|u|}\right)\right)^{\theta}, \quad F_{L}(u)=\mu_{L}|u|^{4 / n}\left(\log \left(R+\frac{1}{|u|}\right)\right)
$$

for some $0 \leq \theta<1, \mu_{S} \in \mathbf{R}$, and $\mu_{L} \in \mathbf{R} . F_{L}$ and $F_{S}$ are called the long- and short-range term, respectively. Here, $R>0$ is a given constant so that for all $0 \leq \tilde{\theta} \leq 1$, and for some sufficiently small constant $0<\delta_{0} \ll 1$, we have

$$
\inf _{t>0}\left(\delta_{0}\left(\log \left(R+\frac{1}{t}\right)\right)^{\tilde{\theta}}-\frac{\tilde{\theta}}{R t+1}\left(\log \left(R+\frac{1}{t}\right)\right)^{\tilde{\theta}-1}\right) \geq 0 .
$$

Remark 1.6. Under this assumption on $\left.R, t^{\delta_{0}} \log (R+1 / t)\right)^{\theta}$ is a monotone increasing functions with respect to $t \geq 0$. This term appears several times, and $\delta_{0}>0$ should be sufficiently small. 
To state the main theorem, we define the following function spaces for $\gamma \in \mathbf{R}$ :

$$
H^{\gamma, 0}:=\left\{\phi \in \mathscr{S}^{\prime}\left(\mathbf{R}^{n}\right) \mid\|\phi\|_{\gamma, 0}:=\left(\int_{\mathbf{R}^{n}}\left(1+|\xi|^{2}\right)^{\gamma}|\mathscr{F}[\phi](\xi)|^{2} d \xi\right)^{1 / 2}<\infty\right\}
$$

and

$$
H^{0, \gamma}:=\left\{\phi \in \mathscr{S}^{\prime}\left(\mathbf{R}^{n}\right) \mid\|\phi\|_{0, \gamma}:=\left(\int_{\mathbf{R}^{n}}\left(1+|x|^{2}\right)^{\gamma}|\phi(x)|^{2} d x\right)^{1 / 2}<\infty\right\},
$$

where $\mathscr{F}$ denotes the Fourier transform. To handle log-like nonlinear terms and consider their asymptotics, the following fractional Leibniz rule is quite important: For some $\gamma>0$, we have

$$
\left\|F_{L}(u) u\right\|_{\gamma, 0} \leq C\left\|F_{L}(u)\right\|_{\infty}\|u\|_{\gamma, 0} .
$$

If $\gamma \in \mathbf{N}$, this inequality can be easily proved. However, to obtain sharp asymptotic estimates, $\gamma$ should be near $1+4 / n$, and therefore the fractional derivative should be calculated. Roy 20, 21 considered loglog nonlinear terms and established the fractional Leibniz rule for such nonlinearities. However, in 20, 21], various technical assumptions (e.g., smoothness for the nonlinearity) are made, and hence this result is difficult to apply to the present model. To overcome this difficulty, we employ the commutator estimate by Li [15, Proposition 3.10] in the following proposition, the proof of which is given in $\S 2$ :

Proposition 1.7. Let $0<\gamma<1+4 / n$ for $n=1,2$ and $0<\gamma \leq 2$ for $n=3$. Then, (9) holds for smooth $u \in H^{\gamma, 0} \cap L^{\infty}\left(\mathbf{R}^{n}\right)$, and the same estimate is true if $F_{L}$ is replaced by $F_{S}$.

Remark 1.8. Unfortunately, lack of smoothness of $F_{L}(u)$ for $n=3$, it is difficult to consider the case where $2<\gamma<1+4 / 3$.

The aim of this paper is to investigate the asymptotic behavior for solution to (1) in $t$. To do this, we first need to show the time-in-local well-posedness for (11), and which can be proven by imitating the proof of Theorem $3.1 \mathrm{in}$ [13. (see Theorem 2.31). In this process, we need to use Proposition 1.7 and 2.2. Then we have that for all $t \in[0, T]$, there is a $C_{T}>0$ such that

$$
\|u(t, \cdot)\|_{\infty} \leq C_{T} \varepsilon^{\prime}\left(1+|t|^{1 / 2} \log (1+|t|)\right)^{-n / 2}
$$

holds. Since $C_{T}$ growth exponentially in $T$, this estimate does not indicate the decay estimate for $\|u(t, \cdot)\|_{\infty}$ in $t$. Hence, in order to obtain the decay estimate for $t \geq T$, we employ the approach by [7.

We now fix $T>0$, denote $C_{T}^{4 / n}$ by $\tilde{C}_{1}$, suppose $\varepsilon^{\prime}>0$ is sufficiently small so that $0<$ $\left(\varepsilon^{\prime}\right)^{4 / 3} \tilde{C}_{1}\left(1+\left|\mu_{L}\right|\right) \ll 1$ and consider the case where $t \geq T$. By virtue of Proposition 1.7 and the approach by [7, we obtain the following theorems:

Theorem 1.9. Let $u_{0} \in H^{\gamma, 0} \cap H^{0, \gamma}$ with $n / 2<\gamma<1+4 / n$ for $n=1,2$ and $n / 2<\gamma \leq 2$ for $n=3$, and $\left\|u_{0}\right\|_{\gamma, 0}+\left\|u_{0}\right\|_{0, \gamma} \leq \varepsilon^{\prime}<\varepsilon$, where $\varepsilon>0$ is sufficiently small. Then, under Assumptions 1.1 and 1.5, there exists a unique global solution to (10) so that $u(t, x) \in C\left(\mathbf{R} ; H^{\gamma, 0} \cap H^{0, \gamma}\right)$ and

$$
\|u(t, \cdot)\|_{\infty} \leq C \varepsilon^{\prime}\left(1+\left|\zeta_{2}(t)\right|\right)^{-n / 2} \leq C \varepsilon^{\prime}\left(1+|t|^{1 / 2} \log (1+|t|)\right)^{-n / 2} .
$$

Theorem 1.10. Under the same assumptions as in Theorem [1.9, and for $u(t, x)$ as in Theorem 1.9, there exist $W \in L^{\infty}\left(\mathbf{R}^{n}\right) \cap L^{2}\left(\mathbf{R}^{n}\right)$ and $\tilde{C}_{1}\left|\mu_{L}\right|\left(\varepsilon^{\prime}\right)^{4 / n}<\alpha<\min (\gamma / 2-n / 4,1)$ such that

$$
\begin{aligned}
& \left\|\mathscr{F}\left(U_{0}(0, t) u(t, \cdot)\right) \exp \left\{i \mu_{L} \int_{r_{0}}^{t} F_{L}\left(\left|\zeta_{2}(\tau)\right|^{-n / 2} \mathscr{F}\left(U_{0}(0, \tau) u(\tau, \cdot)\right)\right) d \tau\right\}-W\right\|_{k} \\
& \leq C\left|\mu_{L}\right| \varepsilon^{\prime}(\log t)^{-\alpha+\tilde{C}_{1}\left|\mu_{L}\right|\left(\varepsilon^{\prime}\right)^{4 / n}}+C\left|\mu_{S}\right| \varepsilon^{\prime}(\log t)^{\theta-1+\tilde{C}_{1}\left|\mu_{L}\right|\left(\varepsilon^{\prime}\right)^{4 / n}}
\end{aligned}
$$


if $\varepsilon^{\prime}$ is sufficiently small so that $\theta+\tilde{C}_{1}\left|\mu_{L}\right|\left(\varepsilon^{\prime}\right)^{4 / n}<1$ and $\tilde{C}_{1}\left|\mu_{L}\right|\left(\varepsilon^{\prime}\right)^{4 / n}<\min (\gamma / 2-n / 4,1)$, where $k$ is 2 or $\infty$. In particular there exists a function $\Phi$ such that

$$
\begin{aligned}
& \left\|u(t)-e^{i F_{L}(|W|)(\log (\log t))+i \Phi} U(t, 0) \mathscr{F}^{-1} W\right\|_{2} \\
& \leq C\left|\mu_{L}\right| \varepsilon^{\prime}(\log t)^{-\alpha+\tilde{C}_{1}\left|\mu_{L}\right|\left(\varepsilon^{\prime}\right)^{4 / n}}+C\left|\mu_{S}\right| \varepsilon^{\prime}(\log t)^{\theta-1+\tilde{C}_{1}\left|\mu_{L}\right|\left(\varepsilon^{\prime}\right)^{4 / n}}
\end{aligned}
$$

holds.

The asymptotic behavior of long-range NLS was investigated in several studies (e.g., Strauss [22], Barab [1], Tsutsumi-Yajima [23], Hayashi-Ozawa [9], Ozawa [19], Ginibre-Ozawa-Velo [5], Ginibre-Velo [6], and Hayashi-Naumkin [7]), and more rigorous analysis has been carried out in, for example, Hayashi-Naumkin-Wang [8], Masaki-Miyazaki-Uriya [17, and Okawamoto-Uriya [18. Regarding NLS with time-dependent harmonic potentials, [2] and Carles-Silva [3] considered general time-dependent harmonic oscillators, which include the present model, and proved various results, such as the unique existence of global-in-time solutions. Subsequently, in [13, which focused on the case $\zeta_{2}(t) \rightarrow \infty$, it was proved that the asymptotic behavior of a solution $v(t, x)$ is

$$
\|v(t, \cdot)\|_{\infty} \leq C\left(1+\left|\zeta_{2}(t)\right|\right)^{-n / 2}
$$

under suitable assumptions on $\sigma(t)$ (Assumption 1.4 in [13]). In particular, if $\sigma(t) t^{2} \rightarrow \sigma_{0} \in$ $[0,1 / 4)$, (10) becomes

$$
\|v(t, \cdot)\|_{\infty} \leq C \varepsilon^{\prime}|t|^{-n\left(1+\sqrt{1-4 \sigma_{0}}\right) / 4}
$$

(see, for instance, [12] and Geluk-Marić-Tomić [4]). We notice that the threshold power of the nonlinearity is $4 /\left(n\left(1+\sqrt{1-4 \sigma_{0}}\right)\right)$. However, the case $\sigma_{0}=1 / 4$, which corresponds to Assumption 1.1, was not considered in [13] because of technical reasons. Accordingly, we consider this case in the present study. This result is new, and the appearance of a log nonlinearity is quite interesting (in the linear case, a similar characterization has been obtained by Ishida-Kawamoto [10]).

Main theorems can be shown by imitating the approach by [7, herein the two norm estimates need to be shown; Lemma 3.1 and

$$
\left\|U_{0}(0, t) u(t)\right\|_{0, \gamma} C \varepsilon^{\prime}(\log t)^{C_{\varepsilon^{\prime}}},
$$

where $t \geq T \gg 1, C>0$ and $C_{\varepsilon^{\prime}}>0$ is sufficiently small. As for the inequality (11), it can be shown with using (91) and the Gronwall inequality, the proof of which is stated below Lemma 3.1. Proof of (9) is stated in $\S 2$, herein we have to deal with the term $\||\nabla| u\|_{\infty}$. Because $1+n / 2>1+4 / n$ for $n=3$, and $u$ may not be included in $H^{\rho, 0}$ with $\rho \geq 1+4 / n$, the Sobolev embedding to $\||\nabla| u\|_{\infty}$ fails for the case $n=3$, which demands the restriction to $\gamma$ in $n=3$. Lemma 3.1 can be show by (11), MDFM-decomposition and the same approach by [7], the proof of which is stated in the last part of $\S 3$.

\section{Preliminaries}

Before proving the main theorem, we prove Proposition 1.7, which is important in [7]. For $n=3$, by considering the case $\gamma=0, \gamma=1$ and $\gamma=2$ and interpolating them one finds Proposition 1.7 for $n=3$. We set $[1+4 / n]<\gamma<1+4 / n$, and let $\tau=\gamma-2$ for $n=2$ and $\tau=\gamma-4$ for $n=1$. We define $\tilde{F}(|u|)=|u|^{4 / n}(\log (R+1 /|u|))^{\tilde{\theta}}, 0 \leq \tilde{\theta} \leq 1$. We only consider the cases $n=2$, as the case $n=1$ is quite similar. 
Proposition 2.1. Let $u \in \mathscr{S}\left(\mathbf{R}^{n}\right)$ with $\mathscr{F}[u] \in C_{0}^{\infty}\left(\mathbf{R}^{n}\right)$. Then, for $0<\delta_{2}=1+4 / n-\gamma$, we have

$$
\|\tilde{F}(|u|) u\|_{\gamma, 0} \leq C\left\||u|^{\delta_{2}}\left(\log \left(R+\frac{1}{|u|}\right)\right)^{\tilde{\theta}}\right\|_{\infty}\|u\|_{\infty}^{1+\tau}\|u\|_{\gamma, 0} .
$$

Proof. We calculate $\langle\nabla\rangle^{\gamma} \tilde{F}(|u|) u$, where $\langle\cdot\rangle=(1+|\cdot|)^{1 / 2}$. We decompose this as $\sum\left\langle D_{j}\right\rangle^{\tau}(1+$ $\left.D_{j}^{2}\right) \tilde{F}(|u|) u$, and we first calculate $D_{j}^{2} \tilde{F}(|u|) u$, where $D_{j}:=\left(-\partial_{j}^{2}\right)^{1 / 2}$. In this process, the terms

$$
\begin{aligned}
& \left\langle D_{j}\right\rangle^{\tau}\left(\left(\log \left(R+\frac{1}{|u|}\right)\right)^{\tilde{\theta}-1}+\left(\log \left(R+\frac{1}{|u|}\right)\right)^{\tilde{\theta}-2}\right)\left(\left(u_{j}\right)^{2}+u_{j j} u\right) \mathcal{O}\left(|u|^{4 / n-1}\right) \\
& \quad+\text { (similar terms ) }
\end{aligned}
$$

and

$$
\left\langle D_{j}\right\rangle^{\tau}\left(\log \left(R+\frac{1}{|u|}\right)\right)^{\tilde{\theta}}\left(u_{j j} u+\left(u_{j}\right)^{2}\right) \mathcal{O}\left(|u|^{4 / n-1}\right)+(\text { similar terms })
$$

appear. In the followings, we only estimate the terms in (14), because the terms in (13) can be handled by the same approach for to handle (14), and find their $L^{2}$ norm can be estimated as $C\|u\|_{\infty}^{4 / n}\|u\|_{\gamma, 0}$ because $(\log (R+1 / t))^{\tilde{\theta}-1}$ and $(\log (R+1 / t))^{\tilde{\theta}-2}$ are uniformly bounded for all $t \geq 0$.

The term associated with $u_{j j} u$ is easy to estimate compared with the term $u_{j}^{2}$, and hence we only consider the latter. By using the identity

$$
\frac{\bar{u}}{|u|}\left(u_{j}\right)^{2}|u|^{-\beta}+|u|^{1-\beta} u_{j j}+\frac{\beta}{1-\beta}|u|^{1-\beta} u_{j j}=\frac{\bar{u}}{|u|} \partial_{j}\left(\frac{u}{|u|^{\beta}} u_{j}\right)+\frac{\beta}{1-\beta} \partial_{j}\left(|u|^{1-\beta} u_{j}\right)
$$

with $\beta=1-\tau$, we obtain

$$
u_{j}^{2} \sim|u| u_{j j}+|u|^{1-\tau} \partial_{j}\left(\mathcal{O}\left(|u|^{\tau}\right) u_{j}\right)
$$

where $\mathscr{F}[u] \in C_{0}^{\infty}\left(\mathbf{R}^{n}\right)$ ensures the boundedness of $|u|^{1-\tau} \partial_{j}\left(\mathcal{O}\left(|u|^{\tau}\right) u_{j}\right)$. Therefore, we estimate

$$
D_{j}^{\tau}\left(\log \left(R+\frac{1}{|u|}\right)\right)^{\tilde{\theta}} \mathcal{O}\left(|u|^{4 / n-\tau}\right) \partial_{j}\left(\mathcal{O}\left(|u|^{\tau}\right) u_{j}\right) .
$$

Let $f=(\log (R+1 /|u|))^{\tilde{\theta}} \mathcal{O}\left(|u|^{4 / n-\tau}\right)$ and $g=D_{j}^{\tau}\left(\mathcal{O}\left(|u|^{\tau}\right) u_{j}\right)$, i.e, $\partial_{j}\left(\mathcal{O}\left(|u|^{\tau}\right) u_{j}\right)=\partial_{j} D_{j}^{-1} \cdot D_{j}^{1-\tau} g$. Then, by the sharp commutator estimate by $\mathrm{Li}$ [15, Proposition 3.10], we obtain

$$
\left\|D_{j}^{\tau}\left(f D_{j}^{1-\tau}\left(\partial_{j} D_{j}^{-1} g\right)\right)-f \partial_{j} g\right\|_{2} \leq C\left\|\partial_{j} f\right\|_{\infty}\left\|\partial_{j} D_{j}^{-1} g\right\|_{2} .
$$

Hence, we have

$$
\begin{aligned}
\left\|D_{j}^{\tau}\left(f \partial_{j}\left(\mathcal{O}\left(|u|^{\tau}\right) u_{j}\right)\right)\right\|_{2} & \leq\left\|f \partial_{j} g\right\|_{2}+C\left\|\partial_{j} f\right\|_{\infty}\|g\|_{2} \\
& \leq\left\|f \partial_{j} g\right\|_{2}+C\left\|\partial_{j} f\right\|_{\infty}\left\|D_{j}^{\tau} \mathcal{O}\left(|u|^{\tau}\right) u_{j}\right\|_{2} .
\end{aligned}
$$

Regarding $\left\|f \partial_{j} g\right\|_{2}$, we first estimate it as

$$
\left\|f \partial_{j} g\right\|_{2} \leq\left\|\mathcal{O}\left(|u|^{4 / n-\tau-1}\right)(\log (R+1 /|u|))^{\tilde{\theta}}\right\|_{\infty}\left\|u \partial_{j} g\right\|_{2} .
$$


Subsequently, we use Proposition 3.10 in [15] and obtain

$$
\left\|u \partial_{j} g\right\|_{2} \leq\left\|D_{j}^{\tau} u D_{j}^{1-\tau}\left(\partial_{j} D_{j}^{-1} g\right)\right\|_{2}+C\left\|\partial_{j} u\right\|_{\infty}\left\|D_{j}^{\tau} \mathcal{O}\left(|u|^{\tau}\right) u_{j}\right\|_{2} .
$$

As

$$
D_{j}^{\tau} u D_{j}^{1-\tau}\left(\partial_{j} D_{j}^{-1} g\right)=-D_{j}^{\tau} \mathcal{O}\left(|u|^{\tau}\right) u_{j}^{2}+D_{j}^{\tau} \partial_{j}\left(\mathcal{O}\left(|u|^{\tau}\right) u u_{j}\right)
$$

for $\delta_{2}=4 / n-\tau-1$, the Gagliardo-Nirenberg interpolation inequality implies

$$
\left\|f \partial_{j} g\right\|_{2} \leq C\left\||u|^{\delta_{2}}\left(\log \left(R+\frac{1}{|u|}\right)\right)^{\tilde{\theta}}\right\|_{\infty}\|u\|_{\infty}^{1+\tau}\|u\|_{\gamma, 0} .
$$

Moreover, as

$$
\begin{aligned}
\left\|\partial_{j} f\right\|_{\infty} & \leq C\left\|\left(\log \left(R+\frac{1}{|u|}\right)\right)^{\tilde{\theta}} \mathcal{O}\left(|u|^{\delta_{2}}\right) \partial_{j} u\right\|_{\infty}+(\text { similar terms }) \\
& \leq C\left\||u|^{\delta_{2}}\left(\log \left(R+\frac{1}{|u|}\right)\right)^{\tilde{\theta}}\right\|_{\infty}\left\|\partial_{j} u\right\|_{\infty},
\end{aligned}
$$

we have

$$
\left\|\partial_{j} f\right\|_{\infty}\|g\|_{2} \leq C\left\||u|^{\delta_{2}}\left(\log \left(R+\frac{1}{|u|}\right)\right)^{\tilde{\theta}}\right\|_{\infty}\|u\|_{\infty}^{1+\tau}\|u\|_{\gamma, 0} .
$$

By combining (15) and (16), Proposition 2.2 is proved.

As $t^{\delta_{2}}(\log (R+1 / t))^{\tilde{\theta}}$ is a monotone increasing function, the assumption $\|u\|_{\infty} \leq C$ implies

$$
\left\||u|^{\delta_{2}}\left(\log \left(R+\frac{1}{|u|}\right)\right)^{\tilde{\theta}}\right\|_{\infty} \leq\|u\|_{\infty}^{\delta_{2}}\left(\log \left(R+\frac{1}{\|u\|_{\infty}}\right)\right)^{\tilde{\theta}} .
$$

Therefore, by Proposition 2.2, we obtain

$$
\|\tilde{F}(|u|) u\|_{\gamma, 0} \leq C\|u\|_{\infty}^{4 / n}\left(\log \left(R+\frac{1}{\|u\|_{\infty}}\right)\right)^{\tilde{\theta}}\|u\|_{\gamma, 0}=C \tilde{F}\left(\|u\|_{\infty}\right)\|u\|_{\gamma, 0} .
$$

As $\tilde{F}$ is also monotone increasing, we have

$$
\|\tilde{F}(|u|)\|_{\infty}=\tilde{F}\left(\|u\|_{\infty}\right) .
$$

Combining (17) and (18), one can prove Proposition 1.7

Thanks to the proposition 1.7 and interpolation theorem, we find the following Proposition.

Proposition 2.2. Let $u, v \in \mathscr{S}\left(\mathbf{R}^{n}\right)$ with $\mathscr{F}[u], \mathscr{F}[v] \in C_{0}^{\infty}\left(\mathbf{R}^{n}\right)$. Then, for the same $\gamma$ in Theorem 1.9.

$$
\|\tilde{F}(|u|) u-\tilde{F}(|v|) v\|_{\gamma, 0} \leq C\left(\|\tilde{F}(|u|)\|_{\infty}+\|\tilde{F}(|v|)\|_{\infty}\right)\|u-v\|_{\gamma, 0} .
$$

By Proposition 1.7 and 2.2, one can prove the existence of a unique local-in-time solution to (11); 
Theorem 2.3. Let $T \gg r_{0}$ be fixed and $u_{0} \in H^{\gamma, 0} \cap H^{0, \gamma}$ with the same $\gamma$ in Theorem 1.9. We assume that $\left\|u_{0}\right\|_{\gamma, 0}+\left\|u_{0}\right\|_{0, \gamma} \leq \varepsilon^{\prime} \leq \varepsilon=\varepsilon(T)$, where $\varepsilon(T)>0$ is sufficiently small compared with $T$. Then, under Assumptions 1.1 and 1.5, there exist a unique local-in-time solution to (11) and $C_{T}>0$ so that $u(t, x) \in C\left([-T, T] ; H^{\gamma, 0} \cap H^{0, \gamma}\right), C_{T} \varepsilon(T)<1 \rightarrow 0$ as $\varepsilon(T) \rightarrow 0$,

$$
\sup _{t \in[-T, T]}\|u(t, \cdot)\|_{\infty} \leq C_{T} \varepsilon^{\prime}\left(1+\left|\zeta_{2}(t)\right|\right)^{-n / 2},
$$

and

$$
\sup _{t \in[-T, T]}\left\|U_{0}(0, t) u(t, \cdot)\right\|_{\gamma, 0} \leq C_{T} \varepsilon^{\prime}
$$

The proof of this theorem is similar to that of Theorem 3.1 in [13, and thus we omit it.

By this theorem, we have $\|u(s, \cdot)\|_{\infty} \leq C_{r_{0}} \varepsilon^{\prime}$ and $\left\|U_{0}(0, t) u(t, \cdot)\right\|_{\gamma, 0} \leq C_{r_{0}} \varepsilon^{\prime}$ for all $t \in$ $\left[-r_{0}, r_{0}\right]$ and for some $C_{r_{0}}>0$. As $T$ is sufficiently large compared with $r_{0}$, one can assume that $0<C_{r_{0}} \ll C_{T}$, and hence without loss of generality, $C_{r_{0}}$ can be denoted by $C$.

\section{Proof of Theorems 1.9 and 1.10}

Herein, we prove Theorems 1.9 and 1.10. Theorem 1.10 can be proved by the arguments in the proof of Theorem 1.9 and the approach in $[7$. Hence, we only prove Theorem [1.9. We first show that $C_{T}$ in Theorem 2.3 can be selected independently of $T$; subsequently, we show Theorem 1.9 by a continuation argument. The second part immediately follows from the first; hence, it suffices to show the first part.

To simplify the proof, we introduce the following lemma, which can be proved by using Lemma 2.11 in [13] and the arguments in the proof of Theorem 4.2 in [13].

Lemma 3.1. For some smooth $u(t)$, we have the estimates

$$
\|u(t)\|_{\infty} \leq C\left|\zeta_{2}(t)\right|^{-n / 2}\left\|\mathscr{F} U_{0}(0, t) u(t)\right\|_{\infty}+C\left|\zeta_{2}(t)\right|^{-n / 2}\left|\frac{\zeta_{1}(t)}{\zeta_{2}(t)}\right|^{\alpha}\left\|U_{0}(0, t) u(t)\right\|_{0, \gamma}
$$

and

$$
\left\|\mathscr{F} U_{0}(0, t) u(t)\right\|_{\infty} \leq C \varepsilon^{\prime}+C \int_{r_{0}}^{t}\left(\left|\mu_{L}\right| I_{1}(\tau)+\left|\mu_{S}\right| I_{2}(\tau)\right) d \tau
$$

with

$$
I_{1}(t)=\left|\frac{\zeta_{1}(t)}{\zeta_{2}(t)}\right|^{\alpha} F_{L}\left(\left|\zeta_{2}(t)\right|^{-n / 2}\left\|U_{0}(0, t) u(t)\right\|_{0, \gamma^{\prime}}\right)\left\|U_{0}(0, t) u(t)\right\|_{0, \gamma}
$$

and

$$
I_{2}(t)=F_{S}\left(\left|\zeta_{2}(t)\right|^{-n / 2}\left\|U_{0}(0, t) u(t)\right\|_{0, \gamma^{\prime}}\right)\left\|U_{0}(0, t) u(t)\right\|_{0, \gamma^{\prime}},
$$

where $t>r_{0}, \gamma^{\prime}>n / 2, \gamma>n / 2+2 \alpha$, and $0<\alpha \leq 1$.

We now prove Theorem [1.9, We first estimate the term $\left\|U_{0}(0, t) u(t)\right\|_{0, \gamma}$. As

$$
\|u(t)\|_{\infty} \leq C_{T} \varepsilon^{\prime}\left(1+\left|\zeta_{2}(t)\right|\right)^{-n / 2}
$$


for $t \in[-T, T]$, and $F_{L}(t)$ and $F_{S}(t)$ are monotone increasing functions on $t \geq 0$, we have

$$
\begin{aligned}
& \left\|U_{0}(0, t) u(t)\right\|_{0, \gamma} \\
& \leq\left\|u_{0}\right\|_{0, \gamma}+C \int_{0}^{t}\left(\left|\mu_{L}\right| F_{L}\left(\|u(s)\|_{\infty}\right)+\left|\mu_{S}\right| F_{S}\left(\|u(s)\|_{\infty}\right)\right)\left\|U_{0}(0, s) u(s)\right\|_{0, \gamma} d s .
\end{aligned}
$$

Then, by the inequality

$$
C \int_{0}^{r_{0}}\left(\left|\mu_{L}\right| F_{L}\left(\|u(s)\|_{\infty}\right)+\left|\mu_{S}\right| F_{S}\left(\|u(s)\|_{\infty}\right)\right)\left\|U_{0}(0, s) u(s)\right\|_{0, \gamma} d s \leq C \varepsilon^{\prime}
$$

we have

$$
\begin{aligned}
& \left\|U_{0}(0, t) u(t)\right\|_{0, \gamma} \\
& \leq C \varepsilon^{\prime}+C C_{T}^{4 / n} \int_{r_{0}}^{t}\left(\varepsilon^{\prime}\right)^{4 / n}\left(1+\left|\zeta_{2}(s)\right|\right)^{-2}\left(\left|\mu_{L}\right| \log \left(R+\frac{\left(1+\left|\zeta_{2}(s)\right|\right)^{n / 2}}{\varepsilon^{\prime} C_{T}}\right)\right. \\
& \left.\quad+\left|\mu_{S}\right|\left(\log \left(R+\frac{\left(1+\left|\zeta_{2}(s)\right|\right)^{n / 2}}{\varepsilon^{\prime} C_{T}}\right)\right)^{\theta}\right)\left\|U_{0}(0, s) u(s)\right\|_{0, \gamma} d s \\
& \leq C \varepsilon^{\prime}+C C_{T}^{4 / n}\left(\varepsilon^{\prime}\right)^{4 / n-1} \int_{r_{0}}^{t}\left(1+\left|\zeta_{2}(s)\right|\right)^{-2}\left(\left|\mu_{L}\right| \varepsilon^{\prime}\left(\log \left(R \varepsilon^{\prime} C_{T}+1+\left|\zeta_{2}(s)\right|^{n / 2}\right)+\log \left(\varepsilon^{\prime} C_{T}\right)\right)\right. \\
& \left.\quad+\left|\mu_{S}\right| \varepsilon^{\prime}\left(\left(\log \left(R \varepsilon^{\prime} C_{T}+1+\left|\zeta_{2}(s)\right|^{n / 2}\right)\right)^{\theta}+\left(\log \left(\varepsilon^{\prime} C_{T}\right)\right)^{\theta}\right)\right)\left\|U_{0}(0, s) u(s)\right\|_{0, \gamma} d s .
\end{aligned}
$$

Clearly, for $0<\tilde{\theta} \leq 1$, we have

$$
\begin{aligned}
& \varepsilon^{\prime}\left(\left(\log \left(R \varepsilon^{\prime} C_{T}+1+\left|\zeta_{2}(s)\right|^{n / 2}\right)\right)^{\tilde{\theta}}+\left(\log \left(\varepsilon^{\prime} C_{T}\right)\right)^{\tilde{\theta}}\right) \\
& \leq C\left(C_{T}^{-1} \varepsilon^{\prime} C_{T}\left(\log \left(\varepsilon^{\prime} C_{T}\right)\right)^{\tilde{\theta}}+\varepsilon^{\prime}\left(\log \left|\zeta_{2}(s)\right|\right)^{\tilde{\theta}}\right) \\
& \leq C\left(C_{T}^{-1}+\varepsilon^{\prime}(\log s)^{\tilde{\theta}}\right),
\end{aligned}
$$

where we use $\left|\log \left(s^{1 / 2} \log s\right)\right| \leq C(\log s+\log \log s) \leq C \log s$ for $s>r_{0} \gg 1$. Then, the inequalities $\left(1+\left|\zeta_{2}(s)\right|\right)^{-2} \leq C s^{-1}(\log s)^{-2}$ and $\int_{r_{0}}^{t}(\cdots) d s \leq C \varepsilon^{\prime}+\int_{0}^{t}(\cdots) d s$ imply $\left\|U_{0}(0, t) u(t)\right\|_{0, \gamma}$

$$
\begin{aligned}
\leq & C \varepsilon^{\prime}+C C_{T}^{4 / n-1}\left(\varepsilon^{\prime}\right)^{4 / n-1}\left(\left|\mu_{S}\right|+\left|\mu_{L}\right|\right) \int_{0}^{t}(1+s)^{-1}(\log (2+s))^{-2}\left\|U_{0}(0, s) u(s)\right\|_{0, \gamma} d s \\
& +C C_{T}^{4 / n}\left(\varepsilon^{\prime}\right)^{4 / n} \int_{0}^{t}(1+s)^{-1}\left(\left|\mu_{L}\right|(\log (2+s))^{-1}+\left|\mu_{S}\right|(\log (2+s))^{-2+\theta}\right)\left\|U_{0}(0, s) u(s)\right\|_{0, \gamma} d s .
\end{aligned}
$$

Hence,

$$
\begin{aligned}
\left\|U_{0}(0, t) u(t)\right\|_{0, \gamma} & \leq C \varepsilon^{\prime} \exp \left\{C_{T}^{4 / n-1}\left(\varepsilon^{\prime}\right)^{4 / n-1}\left(\left|\mu_{S}\right|+\left|\mu_{L}\right|\right)+C_{T}^{4 / n}\left(\varepsilon^{\prime}\right)^{4 / n}\left(\left|\mu_{S}\right|+\left|\mu_{L}\right| \log (\log t)\right\}\right. \\
& \leq C \varepsilon^{\prime} \exp \left\{C_{0, \varepsilon^{\prime}}\left(\left|\mu_{S}\right|+\left|\mu_{L}\right| \log (\log t)\right)\right\}
\end{aligned}
$$

where $C_{0, \varepsilon^{\prime}}=C_{T}^{4 / n}\left(\varepsilon^{\prime}\right)^{4 / n}$ and $0<C_{0, \varepsilon^{\prime}} \ll 1$. 
Estimation of $\|u(t)\|_{\infty}$.

By Lemma 3.1, we have

$$
\begin{aligned}
\|u(t)\|_{\infty} \leq & C \varepsilon^{\prime}\left|\zeta_{2}(t)\right|^{-n / 2}(\log t)^{-\alpha} \exp \left\{C_{0, \varepsilon^{\prime}}\left(\left|\mu_{S}\right|+\left|\mu_{L}\right| \log (\log t)\right)\right\} \\
& +C\left|\zeta_{2}(t)\right|^{-n / 2}\left\|\mathscr{F} U_{0}(0, t) u(t)\right\|_{\infty} .
\end{aligned}
$$

Let now

$$
v(t):=U_{0}(0, t) u(t)
$$

and $\gamma>\gamma^{\prime}$. Then, $\|\cdot\|_{\gamma^{\prime}} \leq\|\cdot\|_{\gamma}$ and (20) imply

$$
\begin{aligned}
\left\|\mathscr{F} U_{0}(0, t) u(t)\right\|_{\infty} \leq & C \varepsilon^{\prime}+\int_{r_{0}}^{t}\left\{\left|\mu_{L}\right|(\log s)^{-\alpha} F_{L}\left(\zeta_{2}(s)^{-n / 2}\|v(s)\|_{0, \gamma}\right)\|v(s)\|_{0, \gamma}\right. \\
& \left.+\left|\mu_{S}\right| F_{S}\left(\zeta_{2}(s)^{-n / 2}\|v(s)\|_{0, \gamma}\right)\|v(s)\|_{0, \gamma}\right\} d s
\end{aligned}
$$

If $f(t)=t^{4 / n}(\log (R+1 / t))^{\tilde{\theta}}$ with $0 \leq \tilde{\theta} \leq 1$ (a monotone increasing function for $t \geq 0$ ) and $C_{1}\left(\varepsilon^{\prime}, \mu_{L}\right):=4\left(C_{0, \varepsilon^{\prime}}\right)\left|\mu_{L}\right| / n$, then (21) implies

$$
\begin{aligned}
& f\left(\left|\zeta_{2}(s)\right|^{-n / 2}\|v(s)\|_{0, \gamma}\right) \\
& \leq C s^{-1}(\log s)^{-2}\left(\varepsilon^{\prime}\right)^{4 / n}(\log s)^{C_{1}\left(\varepsilon^{\prime}, \mu_{L}\right)}\left\{\left(\log \left(R+\frac{s^{n / 4}(\log s)^{n / 2}}{C \varepsilon^{\prime}(\log s)^{C_{0, \varepsilon^{\prime}}\left|\mu_{L}\right|}}\right)\right)^{\tilde{\theta}}\right\} \\
& \leq C s^{-1}(\log s)^{-2+C_{1}\left(\varepsilon^{\prime}, \mu_{L}\right)} \times \begin{cases}\left(\varepsilon^{\prime}\right)^{4 / n-1}, & \left(s(\log s)^{2-C_{1}\left(\varepsilon^{\prime}, \mu_{L}\right)}\right)^{n / 4} \leq 1 /\left(2 C \varepsilon^{\prime}\right), \\
\left(\varepsilon^{\prime}\right)^{4 / n}(\log s)^{\tilde{\theta}}, & \left(s(\log s)^{2-C_{1}\left(\varepsilon^{\prime}, \mu_{L}\right)}\right)^{n / 4} \geq 1 /\left(2 C \varepsilon^{\prime}\right),\end{cases} \\
& \leq C s^{-1}(\log s)^{-2+C_{1}\left(\varepsilon^{\prime}, \mu_{L}\right)+\tilde{\theta}} \varepsilon^{4 / n-1} .
\end{aligned}
$$

Therefore,

$$
\begin{aligned}
&\left\|\mathscr{F} U_{0}(0, t) u(t)\right\|_{\infty} \leq C \varepsilon^{\prime}+C\left|\mu_{L}\right|\left(\varepsilon^{\prime}\right)^{4 / n} \int_{r_{0}}^{t} s^{-1}(\log s)^{-1-\alpha+C_{1}\left(\varepsilon^{\prime}, \mu_{L}\right)+C_{0, \varepsilon^{\prime}}\left|\mu_{L}\right|} \\
&+C\left|\mu_{S}\right|\left(\varepsilon^{\prime}\right)^{4 / n} \int_{r_{0}}^{t} s^{-1}(\log s)^{-2+\theta+C_{1}\left(\varepsilon^{\prime}, \mu_{L}\right)+C_{0, \varepsilon^{\prime}}\left|\mu_{L}\right|} \\
& \leq C \varepsilon^{\prime}
\end{aligned}
$$

where $\tilde{C}_{1}\left(\varepsilon^{\prime}, \mu_{L}\right):=C_{1}\left(\varepsilon^{\prime}, \mu_{L}\right)+C_{0, \varepsilon^{\prime}}\left|\mu_{L}\right|$, and we assume that $-\alpha+\tilde{C}_{1}\left(\varepsilon, \mu_{L}\right)<0$ and $\theta+$ $\tilde{C}_{1}\left(\varepsilon, \mu_{L}\right)<1$, which holds if $\varepsilon^{\prime}$ is sufficiently small because $\tilde{C}_{1}\left(\varepsilon^{\prime}, \mu_{L}\right) \rightarrow 0$ as $\varepsilon^{\prime} \rightarrow 0$.

\section{Models of $\sigma(t)$ satisfying Assumption 1.1}

Herein, we provide simple examples of $\sigma(t)$ satisfying Assumption 1.1. For $\alpha \neq 0$, let

$$
\sigma(t)= \begin{cases}\alpha^{2}, & |t| \leq r_{0}, \\ t^{-2} / 4, & |t|>r_{0} .\end{cases}
$$

Then, $\zeta_{j}(t)$ in (2) can be written as

$$
\zeta_{1}(t)=\cos (\alpha t), \quad \zeta_{2}(t)=\alpha^{-1} \sin (\alpha t)
$$


for $|t| \leq r_{0}$ and

$$
\zeta_{1}(t)=c_{11, \pm} y_{1}(t)+c_{12, \pm} y_{2}(t), \quad \zeta_{2}(t)=c_{21, \pm} y_{1}(t)+c_{22, \pm} y_{2}(t),
$$

where $c_{i j, \pm} \in \mathbf{R}$ are some constants, $y_{1}(t)=|t|^{1 / 2}$, and $y_{2}(t)=|t|^{1 / 2} \log |t|$. By the continuation condition for $\zeta_{j}(t)$ and $\zeta_{j}^{\prime}(t)$, we notice

$$
\begin{aligned}
& c_{11, \pm} r_{0}^{1 / 2}+c_{12, \pm} r_{0}^{1 / 2} \log r_{0}=\cos \left(\alpha\left( \pm r_{0}\right)\right) \\
& c_{21, \pm} r_{0}^{1 / 2}+c_{22, \pm} r_{0}^{1 / 2} \log r_{0}=\alpha^{-1} \sin \left(\alpha\left( \pm r_{0}\right)\right)
\end{aligned}
$$

and

$$
\begin{aligned}
& c_{11, \pm}+c_{12, \pm} \log r_{0}+2 c_{12, \pm}=\mp 2 \alpha r_{0}^{1 / 2} \sin \left(\alpha\left( \pm r_{0}\right)\right), \\
& c_{21, \pm}+c_{22, \pm} \log r_{0}+2 c_{22, \pm}= \pm 2 r_{0}^{1 / 2} \cos \left(\alpha\left( \pm r_{0}\right)\right) .
\end{aligned}
$$

Condition (3) implies $c_{12, \pm}=0$ and $c_{22, \pm} \neq 0$; therefore,

$$
c_{11, \pm}=r_{0}^{-1 / 2} \cos \left(\alpha r_{0}\right)=-2 \alpha r_{0}^{1 / 2} \sin \left(\alpha r_{0}\right)
$$

which implies

$$
\alpha r_{0} \tan \left(\alpha r_{0}\right)=-2
$$

Moreover, by (22) and (23), we obtain

$$
2 c_{22, \pm}= \pm 2 r_{0}^{1 / 2} \cos \left(\alpha r_{0}\right) \mp \alpha^{-1} r_{0}^{-1 / 2} \sin \left(\alpha r_{0}\right)=\frac{ \pm 2 \cos \left(\alpha r_{0}\right)}{\alpha r_{0}^{1 / 2}}\left(\alpha r_{0}-\tan \left(\alpha r_{0}\right)\right) \neq 0
$$

Hence, if $\alpha r_{0}$ satisfies (24) and (25), then $\sigma(t)$ in (2) satisfies Assumption 1.1.

\section{Acknowledgement}

The author is supported by the Grant-in-Aid for Young Scientists \#20K14328 from JSPS.

\section{References}

[1] Barab, J. E.: Nonexistence of asymptotic free solutions for a nonlinear Schrödinger equation, J. Math. Phys., 25, 3270-3273 (1984).

[2] Carles, R.: Nonlinear Schrödinger equation with time dependent potential, Commun. Math. Sci., 9, 937-964 (2011).

[3] Carles, R., Silva, J. D.: Large time behavior in nonlinear Schrödinger equation with time dependent potential, Comm. Math. Sci., 13, 443-460 (2015).

[4] Geluk, J. L., Marić, V., Tomić, M.: On regularly varying solutions of second order linear differential equations, Differential and Integral Equ., 6, 329-336, (1993).

[5] Ginibre, J., Ozawa, T., Velo, G.: On the existence of the wave operators for a class of nonlinear Schrödinger equations, Ann. de l'L.H.P. Phys. théorique, 60 211-239 (1994).

[6] Ginibre, J., Velo, G.: Scattering theory in the energy space for a class of nonlinear Schrödinger equations, J. Math. Pure. Appl., 64, 363-401 (1985). 
[7] Hayashi, N., Naumkin, I. P.: Asymptotics for large time of solutions to the nonlinear Schrödinger and Hartree equations, American J. of Math., 120, 369-389 (1998).

[8] Hayashi, N., Naumkin, I. P., Wang, H.: Modified wave operators for nonlinear Schrödinger equations in lower order Sobolev spaces, J. Hyperbolic Diff. Eqn., 8, 759 $775(2011)$

[9] Hayashi, N., Ozawa, T.: Scattering theory in the weighted $L^{2}\left(\mathbb{R}^{n}\right)$ spaces for some Schrödinger equations, Ann. Inst. H. Poincaré, Phys. Théor., 48, 17-37 (1988)

[10] Ishida, A., Kawamoto, M.: Critical scattering in a time-dependent harmonic oscillator, J. Math. Anal. Appl., 492, 124475 (9 pages), (2020).

[11] Kawamoto, M.: Mourre theory for time-periodic magnetic fields, J. Funct. Anal., 277, 1-30 (2019).

[12] Kawamoto, M., Yoneyama, T.: Strichartz estimates for harmonic potential with timedecaying coefficient, J. Evol. Eqn. 18, 127-142 (2017) .

[13] Kawamoto, M., Muramatsu, R.: Asymptotic behavior of solutions to nonlinear Schrödinger equations with time-dependent harmonic potentials, J. Evol. Eqn., 21, 699-723 (2021).

[14] Korotyaev, E. L.: On scattering in an external, homogeneous, time-periodic magnetic field, Math. USSR-Sb., 66, 499-522 (1990).

[15] Li, D.: On Kato-Ponce and fractional Leibniz, Rivista Math. Iber., 35, 23-100 (2019).

[16] Masaki, S., Miyazaki, H.: Long range scattering for nonlinear Schrödinger equations with critical homogeneous nonlinearity, SIAM, J. Math. Anal. 50, 3251-3270 (2018).

[17] Masaki, S., Miyazaki, H., Uriya, K.: Long-range scattering for nonlinear Schrödinger equations with critical homogeneous nonlinearity in three space dimensions, Transaction of the A.M.S., 371, 7925-7947 (2019).

[18] Okamoto, M., Uriya, K.: Final state problem for the nonlocal nonlinear Schrödinger equation with dissipative nonlinearity, Diff., Eqn., and Appl., 11, 481-494 (2019).

[19] Ozawa, T.: Long range scattering for nonlinear Schrödinger equations in one space dimension, Com. Math. Phys., 139, 479-493 (1991).

[20] Roy, T.: Scattering above energy norm of solutions of a loglog energy-supercritical Schrödinger equation with radial data, J. Diff. Eqn., 250, 292-319 (2011).

[21] Roy, T.: On Jensen-type inequalities for nonsmooth radial scattering solutions of a $\log \log$ energy-subcritical Schrödinger equation, Int. Math. Res. Not., IMRN 2020, 2501-2541 (2020).

[22] Strauss, W. A.: Nonlinear scattering theory, scattering theory in Math. Physics, Reidel, Dordrecht, 1974, pp. 53-78.

[23] Tsutsumi, Y., Yajima, K.: The asymptotic behavior of nonlinear Schrödinger equations, Bull. Amer. Math. Soc., 11, 186-188 (1984). 